\title{
Mitochondrial Membrane
}

National Cancer Institute

\section{Source}

National Cancer Institute. Mitochondrial Membrane. NCI Thesaurus. Code C25937.

The double membrane structure surrounding the mitochondrion and separating it from the cell cytoplasm. The two membranes are separated by an intermembrane space. The electron transport chain resides in the inner mitochondrial membrane. 